\title{
Element abundances in the metal-rich open cluster NGC $6253^{\star, \star \star}$
}

\author{
P. Sestito ${ }^{1,2}$, S. Randich ${ }^{1}$, and A. Bragaglia ${ }^{3}$ \\ 1 INAF-Osservatorio Astrofisico di Arcetri, Largo E. Fermi 5, 50125 Firenze, Italy \\ e-mail: [sestito;randich] @arcetri.astro.it \\ 2 INAF-Osservatorio Astronomico "G.S. Vaiana" di Palermo, Piazza del Parlamento 1, 90134 Palermo, Italy \\ 3 INAF-Osservatorio Astronomico di Bologna, Via C. Ranzani 1, 40127 Bologna, Italy \\ e-mail: angela.bragaglia@oabo.inaf.it
}

Received 26 October 2006 / Accepted 2 January 2007

ABSTRACT

\begin{abstract}
Context. We have carried out a big FLAMES survey of 10 Galactic open clusters aiming at different goals. One of them is the determination of chemical abundances, to put constraints on the radial metallicity gradient in the disk and its evolution. One of the sample clusters is the very metal-rich NGC 6253.

Aims. We have obtained UVES high resolution spectra of seven candidate cluster members (from the turn off up to the red clump) with the goal of determining the chemical composition of NGC 6253 and of investigating its origin and role in the interpretation of the radial metallicity gradient in the disk.

Methods. Equivalent width analysis and spectral synthesis were performed using MOOG and Kurucz model atmospheres.

Results. We derived abundances of $\mathrm{Fe}, \alpha$-, and Fe-peak elements, the light element $\mathrm{Na}$, and the s-process element Ba. Excluding two likely non-members and the clump giant, whose metallicity from equivalent widths is overestimated, we find an average $[\mathrm{Fe} / \mathrm{H}]=+0.36 \pm 0.07(\mathrm{rms})$ for the cluster. For most of the other elements we derive solar abundance ratios.
\end{abstract}

Key words. stars: abundances - Galaxy: evolution - Galaxy: disk - open clusters and associations: individual: NGC 6253 Galaxy: abundances - open clusters and associations: general

\section{Introduction}

The advent of new observational capabilities in the last years allowed a steady improvement in the field of chemical abundances in astrophysical objects studied through high resolution spectroscopy. In particular, it is now possible to derive precise element abundances in old and distant Galactic open clusters, allowing us to address different issues such as the origin of the clusters themselves, the Galactic radial metallicity gradient, and the formation and evolution of the Galactic disk (see, e.g., Friel 2006 and references therein). At the same time, the abundances of other species, such as $\alpha$ - and Fe-peak elements, or the s- and r-process elements, and their ratios to $\mathrm{Fe}$, are crucial to obtain insights into the role of stars with different masses and evolutionary lifetimes in the heavy element enrichment of the interstellar medium. This, through comparison with Galactic enrichment models, permits us to put constraints on the initial mass function and star formation history during the early phases of disk evolution.

In this context, we carried out a VLT/FLAMES program on a sample of 10 open clusters (Randich et al. 2005). One of the main goals of this project is the determination of the cluster metallicity and chemical composition through the analysis of UVES spectra of evolved members (Sestito et al. 2006 - hereafter Paper I). We focus here on the $\sim 3$ Gyr old cluster

^ Based on observations collected at ESO telescopes under program 072.D-0550(A).

$\star \star$ Table 3 is only available in electronic form at the CDS via anonymous ftp to cdsarc.u-strasbg.fr (130.79.128.5) or via http://cdsweb.u-strasbg.fr/cgi-bin/qcat?J/A+A/465/185
NGC 6253; this object, located towards the Galactic center, is one of the most interesting clusters in our sample, since it has a metallicity considerably higher than solar. Twarog et al. (2003) suggested that NGC 6253 might be the most metal-rich object in the Galaxy. From photometric indices, they found $[\mathrm{Fe} / \mathrm{H}] \sim$ +0.7 , while from comparison with isochrones they concluded that $\alpha$-enhanced isochrones provided the best fit, indicating a $[\mathrm{Fe} / \mathrm{H}]$ closer to +0.4 . Besides the study by Twarog et al. (2003) various photometric surveys of this cluster were carried out in the last $~ 10$ years (Bragaglia et al. 1997; Piatti et al. 1998; Sagar et al. 2001); most of them yielded very similar values of reddening, age, and distance, namely $E(B-V) \simeq 0.20$, age $\sim 3 \mathrm{Gyr}$, and $(m-M)_{0}=10.9-11.0(d \sim 1.8 \mathrm{kpc})$. The first spectroscopic determination of the metallicity of NGC 6253 was carried out by Carretta et al. (2000), who found $[\mathrm{Fe} / \mathrm{H}]=+0.36 \pm 0.20$. A more recent analysis (Carretta et al., submitted) based on better quality spectra of five red clump stars favors a higher metallicity $([\mathrm{Fe} / \mathrm{H}]=+0.46)$.

Besides NGC 6253, a few other open clusters with metallicity higher than solar are confirmed by spectroscopic means (see Randich 2007 and references therein). Among them we mention NGC 6475 and Hyades (ages 250 and $600 \mathrm{Myr}$, $[\mathrm{Fe} / \mathrm{H}]=+0.14$ and +0.13 , respectively: see Sestito et al. 2003 and Boesgaard \& Friel 1990), Praesepe ( 600 Myr, $[\mathrm{Fe} / \mathrm{H}]=$ +0.25 ; Pace et al., in preparation), the $\sim 1$ Gyr old NGC 6134 $([\mathrm{Fe} / \mathrm{H}]=+0.15$, Carretta et al. 2004), and the 2 Gyr old IC 4651 $([\mathrm{Fe} / \mathrm{H}]=+0.10$ : Pasquini et al. 2004; Carretta et al. 2004). The most noticeable metal-rich cluster is the very old ( 8-9 Gyr) NGC 6791, recently investigated by Origlia et al. (2006), Carraro et al. (2006), and Gratton et al. (2006), who derive metallicities 
Table 1. Observation log of NGC 6253.

\begin{tabular}{llllll}
\hline \hline Date & UT $_{\text {beginning }}$ & $\begin{array}{l}\text { Exposure time } \\
(\mathrm{s})\end{array}$ & Configuration & Grating & No. of stars \\
\hline $2004-04-07$ & 073140.549 & 2595 & A & CD3 & 7 \\
$2004-04-08$ & 073950.833 & 2595 & B & CD3 & 6 \\
$2004-04-06$ & 083434.455 & 2595 & A & CD4 & 7 \\
$2004-04-07$ & 082740.831 & 2595 & A & CD4 & 7 \\
$2004-04-08$ & 083719.858 & 2595 & B & CD4 & 6 \\
\hline
\end{tabular}

of $[\mathrm{Fe} / \mathrm{H}]=+0.35,+0.39$, and +0.47 , respectively (from giant stars).

The origin of metal-rich disk clusters is puzzling, especially in the case of old ones, since the classical view of Galactic evolution predicts an over-time enrichment of the interstellar medium, and as a consequence only the youngest stars should have metallicities higher than solar. Nevertheless, other old and metal-rich stellar populations exist, such as bulge field stars (Fulbright et al. 2006,2007 ) and objects in the solar neighborhood with kinematics and metallicities more similar to those of the bulge, in spite of their position (Castro et al. 1997; Pompeia et al. 2003). The high metallicity of stars in the center of the Galaxy can be explained with an early enrichment of that region, while the presence of old metal-rich stars/open clusters in the disk is more puzzling. One hypothesis about the origin of metal-rich open clusters is that they were born in the inner side of the Galaxy, close to the bulge, where the metal enrichment occurred early and rapidly, and then they moved outwards in the disk. Alternatively, they might have been born in an external environment and then captured by our Galaxy, as discussed by Carraro et al. (2006) for NGC 6791, although a very recent paper on this cluster excludes this possibility (Bedin et al. 2006). Finally, the simplest explanation is that metal-rich clusters originated in the disk itself, in a region characterized by faster enrichment. The element abundance distribution of metal-rich open clusters is very useful to put constraints on their origin.

Independently of their origin, the very existence of metalrich open clusters provides ideal samples to investigate other topics, such as the dependence of light element depletion on chemical composition, or planet formation and evolution. In particular, it is now well ascertained that stars hosting giant planets are more metal-rich than stars not harboring planetary systems (e.g., Santos 2006 and references therein). Metal-rich clusters thus represent very good targets in which to search for planetary systems, although photometric searches for transiting planets have not been successful so far (but have shown feasibility - see, e.g., Paulson et al. 2004a,b for the Hyades; Mochejska et al. 2005 for NGC 6791).

We present here a new high resolution spectroscopic investigation of NGC 6253; with respect to Carretta et al. (2000, 2007, submitted) our sample covers a much wider region in the color-magnitude diagram (CMD), including not only a red clump member, but also turn off (TO) and subgiant/red giant branch (SGB, RGB) stars. Since, as we will show in the paper, at very high metallicities the Fe content of clump stars derived with equivalent width analysis might be overestimated - due to heavy line blending - the analysis of hotter TO and RGB stars should in principle provide more reliable results. The paper is organized as follows: in Sect. 2 we describe the sample and data reduction, while Sect. 3 is dedicated to the method of analysis and estimate of uncertainties. In Sect. 4 we report our results, checking the validity of the metallicity scale. The results are then discussed in Sect. 5, and summarized in Sect. 6.

\section{Observations and data reduction}

The spectra of the NGC 6253 sample presented in this paper were collected with FLAMES on VLT/UT2 (Pasquini et al. 2000), using the fiber link to UVES with a spectral resolution of $R=47000$. The GIRAFFE fibers were instead used for collecting spectra of a large number of main sequence stars, with the goal of investigating lithium abundances and radial velocities (Randich et al., in preparation). The observations were carried out in service mode during April 2004, with two FLAMES configurations, using two different gratings (CD3 and CD4, covering the wavelength ranges $\sim 4750-6800 \AA$ and $6600-10600 \AA$, respectively) for each of them. We used two configurations to maximize the number of objects observed with GIRAFFE; as a consequence, the UVES pointings also changed. More in detail, the two configurations differ for the number of stars observed (seven and six stars were observed with UVES in configurations A and B, respectively) and for the correspondence between fiber and object. Since all the six stars observed with UVES in configuration $\mathrm{B}$ are in common with configuration $\mathrm{A}$, we observed seven stars in total. Table 1 gives the log of observations for the cluster. Data reduction was carried out by ESO personnel using the dedicated pipeline, and we analyzed the 1-d, wavelength calibrated spectra using standard IRAF ${ }^{1}$ packages. The contamination by atmospheric telluric lines was taken into account by performing a correction on the spectra with the task TELLURIC in IRAF via a comparison with early-type stars observed with UVES during another run. Background subtraction was carried out, as customary, using one fiber dedicated to the sky.

We report the target stars in Table 2, adopting the ID numbers from the EIS survey (Momany et al. 2001; Col. 1); since we used the photometry by Bragaglia et al. (1997; Cols. 6 and 7), we also list for completeness their IDs in Col. 2. The only star not included in the study by Bragaglia et al. (1997) is 105495, for which we adopted the EIS photometry calibrated to Bragaglia et al. (1997). As mentioned in Sect. 1, NGC 6253 was also investigated by Twarog et al. (2003); therefore we provide a crossidentification with their numbering system (Col. 3). The signalto-noise $(S / N)$ ratios reported in Col. 10 have been measured in the spectral regions around $5600 \AA$ and $6300 \AA$.

We measured radial velocities $(R V)$ with RVIDLINES using several tens of metallic lines on each single spectrum, and subsequently we combined multiple spectra. The heliocentric $R V \mathrm{~s}$ (Col. 9 of Table 2) have uncertainties of $\sim 1 \mathrm{~km} \mathrm{~s}^{-1}$ with the exception of star 023501 for which the error is almost $3 \mathrm{~km} \mathrm{~s}^{-1}$. The mean $R V$ of the whole sample is $-29.85 \pm 4.55 \mathrm{~km} \mathrm{~s}^{-1}: R V \mathrm{~s}$ of stars 069360 and 022182 deviate from this value by more than $1 \sigma$; therefore we consider them non-members, although we cannot exclude that they are binary cluster members. If we exclude

1 IRAF is distributed by the National Optical Astronomical Observatories, which are operated by the Association of Universities for Research in Astronomy, under contract with the National Science Foundation. 
Table 2. Data for NGC 6253. $\mathrm{ID}_{\mathrm{B} 97}$ and $B V$ photometry (non corrected for reddening) are from Bragaglia et al. (1997) except for star 105495, see text; we also report $\mathrm{ID}_{\mathrm{EIS}}$, used through out the paper, and a cross-identification with Twarog et al. (2003). The number of exposures for each star is intended as a number of pointings with the same cross-disperser.

\begin{tabular}{|c|c|c|c|c|c|c|c|c|c|c|}
\hline $\begin{array}{l}\text { Star } \\
\text { ID }\end{array}$ & $\begin{array}{l}\text { Star } \\
\text { ID }_{\text {B97 }}\end{array}$ & $\begin{array}{l}\text { Star } \\
\mathrm{ID}_{\mathrm{T} 03} \\
\end{array}$ & $\overline{\overline{\mathrm{RA}}}$ & $\overline{\overline{\text { Dec }}}$ & $\overline{\bar{V}}$ & $\overline{\bar{B}}$ & $\begin{array}{l}\text { No. exp. } \\
\text { CD3/CD4 }\end{array}$ & $\begin{array}{l}R V \pm \mathrm{rms} \\
\left(\mathrm{km} \mathrm{s}^{-1}\right)\end{array}$ & 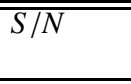 & Notes \\
\hline 069885 & 3053 & 175 & 165852.311 & -524140.76 & 14.400 & 15.210 & $2 / 3$ & $-28.81 \pm 1.30$ & $70-80$ & TO, M \\
\hline 023501 & 2225 & 160 & 165853.250 & -524243.32 & 14.330 & 15.192 & $2 / 3$ & $-33.49 \pm 2.87$ & $65-90$ & TO, NM? \\
\hline 069360 & 2542 & 73 & 165907.044 & -524221.84 & 13.229 & 14.520 & $1 / 2$ & $-21.07 \pm 0.59$ & $100-120$ & RGB, NM \\
\hline 022182 & 1556 & 127 & 165915.518 & -524333.11 & 13.865 & 15.094 & $2 / 3$ & $-35.51 \pm 0.77$ & $90-110$ & SGB/RGB, NM \\
\hline 023498 & 2253 & 133 & 165921.358 & -524243.18 & 13.933 & 14.886 & $2 / 3$ & $-30.13 \pm 1.01$ & $65-100$ & SGB, M \\
\hline 024707 & 3138 & 97 & 165912.924 & -524135.76 & 13.548 & 14.726 & $2 / 3$ & $-30.58 \pm 0.65$ & $75-100$ & SGB/RGB, M \\
\hline 105495 & - & 52 & 165851.192 & -523657.47 & 12.700 & 14.110 & $2 / 3$ & $-29.33 \pm 0.84$ & $120-140$ & clump, $\mathrm{M}$ \\
\hline
\end{tabular}

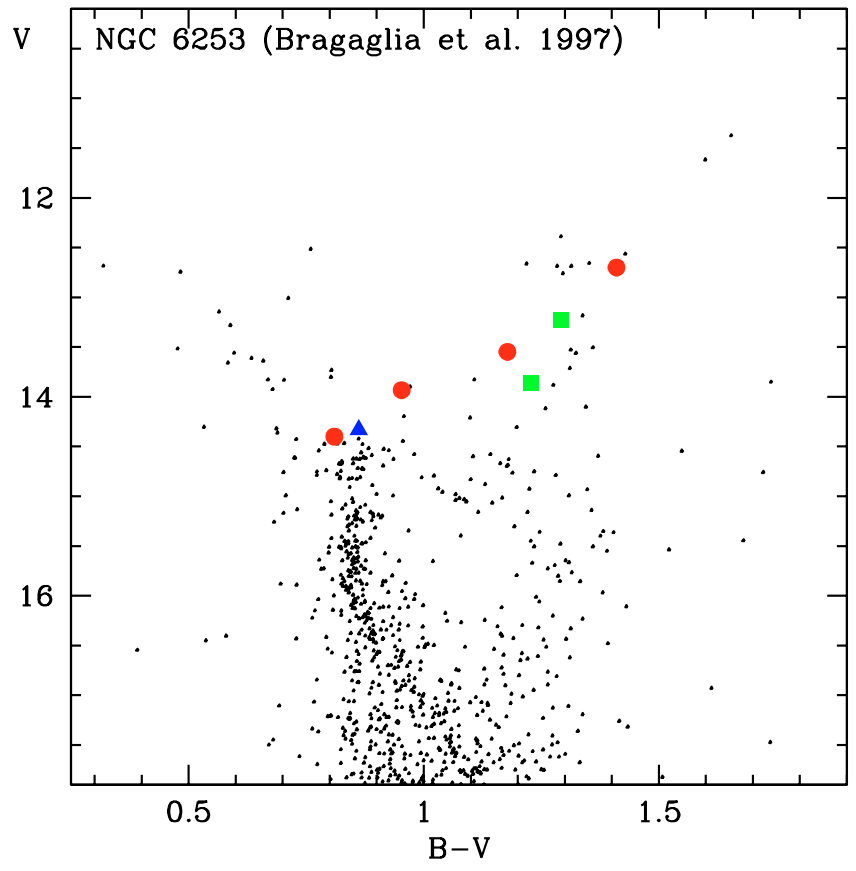

Fig. 1. Color-magnitude diagram for NGC 6253. The observed stars are evidenced by circles (members), triangles (the radial velocity doubtful member, but with metallicity consistent with membership), and squares (non-members).

these two stars, we obtain $\langle R V\rangle=-30.47 \pm 1.82 \mathrm{~km} \mathrm{~s}^{-1}$ for the remaining 5 objects. However, star 023501 also has an $R V$ slightly deviating from the two averages above (by $\sim 3 \mathrm{~km} \mathrm{~s}^{-1}$ ), therefore we will provisionally consider it as a doubtful member (see below and Sect. 4). By computing the average radial velocity considering only the 4 stars that can be safely classified as members, we have $\langle R V\rangle=-29.71 \pm 0.79 \mathrm{~km} \mathrm{~s}^{-1}$. Figure 1 shows the CMD of the cluster, where the selected stars are marked using different symbols. Note that the positions in the CMD of stars with radial velocity deviating from the mean are consistent with membership (in particular that of the doubtful member 023501). Notes on the evolutionary status and membership of the stars are shown in Col. 11 of Table 2. Finally, in Fig. 2 we show the spectra (referred to $R V=0$ ) in the wavelength region around the $\mathrm{H} \alpha$ feature for all the observed stars.

\section{Analysis}

\subsection{Line lists and equivalent widths}

The analysis of chemical abundances was performed by means of equivalent widths ( $E W \mathrm{~s}$ ) using an updated version (2006)

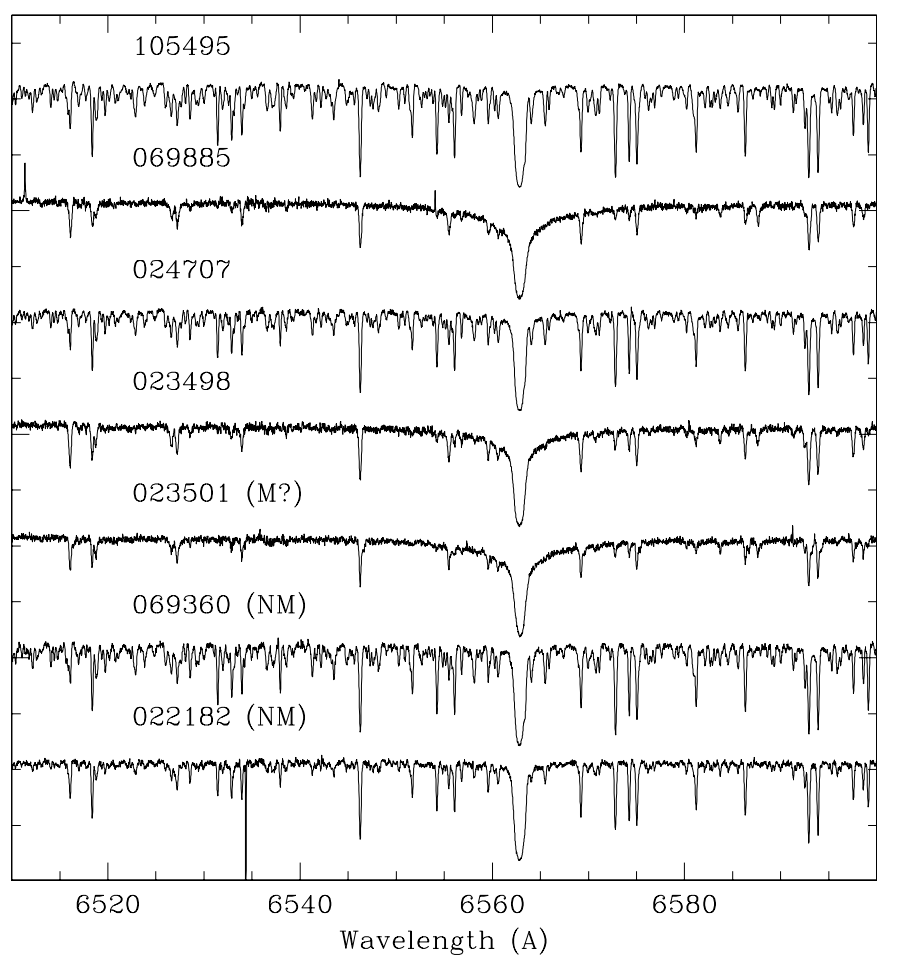

Fig. 2. NGC 6253 sample spectra in the wavelength region at 6500-6600 ̊.

of the package MOOG (Sneden 1973) ${ }^{2}$ and using model atmospheres by Kurucz (1993). MOOG works under the assumption of local thermodynamic equilibrium (LTE). Solar abundances of $\mathrm{Fe}$ and other elements ( $\mathrm{Na}, \mathrm{Mg}, \mathrm{Si}, \mathrm{Ca}, \mathrm{Ti}, \mathrm{Cr}, \mathrm{Ni}$, $\mathrm{Ba}$ ) were derived to determine the zero point of the metallicity scale. The line lists adopted for the Sun and for evolved stars were retrieved from Gratton et al. (2003) and are described in Paper I and Bragaglia et al. (in preparation). We recall from Paper I that when available we adopted collisional damping coefficients from Barklem et al. (2000), otherwise we considered the coefficients by Gratton et al. (2003), or, for a few lines, the classical Unsöld (1955) approximation. For the Sun we obtain $\log n(\mathrm{Fe} \mathrm{I})=7.49 \pm 0.04$ (standard deviation, or rms) using $T_{\text {eff }}=5779 \mathrm{~K}, \log g=4.44$, and $\xi=0.8 \mathrm{~km} \mathrm{~s}^{-1}$.

The spectra were normalized using CONTINUUM in IRAF, dividing the spectra in small regions (50 $)$ ) and visually checking the output; $E W \mathrm{~s}$ for the various lines were measured with SPECTRE (developed and maintained by C. Sneden) by Gaussian fitting of the line profiles. We provide the $E W$ s for each

\footnotetext{
${ }^{2}$ http://verdi.as.utexas.edu/
} 
Table 4. Stellar parameters and Fe abundances for stars in NGC 6253. Numbers in parenthesis in Col. 6 are [Fe/H] estimated by spectral synthesis.

\begin{tabular}{lllllllll}
\hline \hline Star & $\begin{array}{l}T_{\text {eff phot }} \\
(\mathrm{K})\end{array}$ & $\log g_{\text {phot }}$ & $\begin{array}{l}T_{\text {eff spec }} \\
(\mathrm{K})\end{array}$ & $\begin{array}{l}\xi \\
\left(\mathrm{km} \mathrm{s}^{-1}\right)\end{array}$ & {$[\mathrm{Fe} / \mathrm{H}]$} & $\sigma_{1}$ & $\sigma_{\text {tot }}$ & Membership \\
\hline 069885 & 6032 & 3.84 & 6200 & 1.27 & $+0.45(+0.35)$ & 0.09 & 0.11 & $\mathrm{M}$ \\
023501 & 5842 & 3.75 & 6050 & 0.90 & +0.29 & 0.09 & 0.11 & $\mathrm{M} ?$ \\
023498 & 5509 & 3.44 & 5630 & 1.30 & +0.32 & 0.09 & 0.11 & $\mathrm{M}$ \\
024707 & 4972 & 3.05 & 4940 & 1.16 & +0.39 & 0.09 & 0.11 & $\mathrm{M}$ \\
105495* & 4520 & 2.46 & 4450 & 1.23 & $+0.49(+0.35)$ & 0.10 & 0.13 & $\mathrm{M}$ \\
Average Fe & & & & & $+0.39(+0.34)$ & & $0.08(0.04)(\mathrm{rms})$ & \\
\hline Likely non-members & & & & & & & & \\
06360 & 4741 & 2.81 & 4680 & 1.20 & +0.48 & 0.10 & 0.12 & $\mathrm{NM}$ \\
022182 & 4865 & 3.13 & 4770 & 1.20 & +0.12 & 0.07 & 0.09 & $\mathrm{NM}$ \\
\hline
\end{tabular}

* Note: the initial parameters of the clump star have been computed from EIS magnitudes calibrated to Bragaglia et al. (1997, see Table 2). We also performed a test using the Strömgren photometry by Twarog et al. (2003) reported to the $U B V$ system. In this case we found $T_{\text {eff phot }}=4666 \mathrm{~K}$ and $\log g=2.58$, and, from the spectroscopic analysis, $T_{\text {eff spec }}=4500 \mathrm{~K}, \xi=1.23 \mathrm{~km} \mathrm{~s}^{-1},[\mathrm{Fe} / \mathrm{H}]=+0.51(\log g=2.58)$, which agree within the errors with the values reported in Table 4.

star in Table 3 (available only in electronic format the CDS): the first two columns list the wavelength and the element, and the others show the corresponding $E W$ for each star. Note that the definition of the continuum and the determination of the $E W \mathrm{~s}$ are a very critical step for our stars, since they are rather cool objects with low gravity and with an exceptionally high metallicity. As a consequence, several lines can be affected by strong blending (see below also).

\subsection{Stellar parameters}

Initial effective temperatures ( $\left.T_{\text {eff }}\right)$ and gravities were estimated from photometry. In the case of giant stars we used the $B-V$ vs. $T_{\text {eff }}$ calibration by Alonso, Arribas, $\&$ Martinez-Roger (1999) for giants, while for TO stars we adopted the calibration by Alonso et al. (1996), based on a large sample of dwarfs. Surface gravities were derived using the expression $\log g=\log \left(M / M_{\odot}\right)+$ $0.4\left(M_{\text {bol }}-M_{\text {bol } \odot}\right)+4 \cdot \log \left(T_{\text {eff }} / T_{\text {eff } \odot}\right)+\log g_{\odot}$, where $M$ is the mass and $M_{\text {bol }}$ the bolometric magnitude (with $M_{\text {bol } \odot}=4.72$ ). Bolometric corrections for giants were derived following Alonso et al. (1999), while those for the two TO stars were retrieved from Johnson (1966) and are close to 0. We adopted the most recent cluster parameters by Bragaglia \& Tosi $(2006),(m-M)_{0}=$ 11.0, $E(B-V)=0.23$, and an age of $3 \mathrm{Gyr}$, which, using $Z=0.05$ isochrones, corresponds to masses $M \sim 1.32 M_{\odot}$ at the TO and $\sim 1.40 M_{\odot}$ at the clump. We assumed $M=1.40 M_{\odot}$ for all the stars, since uncertainties $\lesssim 0.1 M_{\odot}$ translate into differences of 0.03 dex or lower in surface gravities, which are well below the random errors and do not affect the metallicities derived from Fe I lines. Note that the calibrations by Alonso et al. $(1996 ; 1999)$ are valid up to a metallicity of $[\mathrm{Fe} / \mathrm{H}]=+0.2$, while the cluster should have a higher Fe content; nevertheless, the weak dependence of the $T_{\text {eff }}$ on $B-V$ colors suggests that the error is small, and in any case the photometric temperatures are used only as initial parameters and are optimized during the spectroscopic analysis.

In Paper I the microturbulent velocities were derived using the relationship by Carretta et al. (2004), based on the optimization of Fe abundance as a function of theoretically expected $E W \mathrm{~s}$ for the given lines (see the quoted references for further details). The formula cannot be safely applied for NGC 6253, since it was based on a sample of giants in clusters with metallicities closer to solar; in this case we deal instead with a very metal-rich cluster and also with TO stars. Therefore, we only used the microturbulences by Carretta et al. (2004) as starting values; then, we optimized them by minimizing the slope of $\log n(\mathrm{Fe})$ I vs. the observed EWs; when plotting Fe abundances as a function of expected $E W \mathrm{~s}$, we obtain a rather small trend (i.e., the slope of the relationship is smaller than its error), suggesting that the two methods of analysis are in fair agreement.

As is customary, final effective temperatures were derived during the analysis (after $1 \sigma$ clipping) by minimizing the trend of $\log n(\mathrm{Fe} \mathrm{I})$ vs. the excitation potential $(E P)$; as for surface gravities, we cannot derive them from the ionization equilibrium condition (i.e., the assumption that the difference between Fe I and Fe II abundances in the stars analyzed should be similar to that found for the Sun) since the few lines of ionized Fe are strongly affected by blending. On the other hand, since in most cases spectroscopic temperatures are in good agreement with photometric ones and the distance is well known, we decided to adopt the photometric $\log g$ values and left them unchanged during the analysis. The only stars for which the spectroscopic and photometric $T_{\text {eff }}$ differ by a rather significant amount ( $\left.200 \mathrm{~K}\right)$ are the TO stars; note, however, that changing the $T_{\text {eff }}$ by $\pm 200 \mathrm{~K}$ would imply a variation of $\sim \pm 0.1 \mathrm{dex}$ in $\log g$, consistent with the errors (see Sect. 3.3). For the reasons mentioned above, we retained only neutral $\mathrm{Fe}$ features in the analysis. In Table 4 we list the photometric and spectroscopic $T_{\text {eff }}$ (Cols. 2 and 4), the adopted surface gravity (photometric, Col. 3), and the spectroscopic microturbulence (Col. 5) for each star.

\subsection{Errors}

The major source of random uncertainties affecting element abundances derive from errors in $E W$ s and uncertainties in stellar parameters; systematic uncertainties come from biases due to the method of analysis adopted and from errors in the line list (i.e., possible blendings and oscillator strengths). The errors in abundances related to $E W \mathrm{~s}$ are given in good approximation by the standard deviation (rms) around the mean abundance derived from individual lines for each star, call it $\sigma_{1}$. This rms also includes the errors related to atomic parameters: $\log g f$ values were taken from the literature, thus we cannot give a precise estimate of the effect of their uncertainties; however, since our abundance scale is directly related to solar abundances and the line list used for cluster stars is very similar to that adopted for the Sun, we can assume that internal errors due to uncertainties in the atomic parameters are minimized. Note that when the 
Table 5. Sensitivities of abundances $(\log n(\mathrm{X}))$ to variations in the atmospheric parameters for TO and clump stars in NGC 6253.

\begin{tabular}{cccc}
\hline \hline$\Delta$ & $\sigma_{T \text { eff }}$ & $\sigma_{\log g}$ & $\sigma_{\xi}$ \\
& $\Delta T_{\text {eff }}= \pm 70 \mathrm{~K}$ & $\Delta \log g= \pm 0.25 \mathrm{dex}$ & $\Delta \xi= \pm 0.10 \mathrm{~km} \mathrm{~s}^{-1}$ \\
\hline 069885 & & & \\
$\mathrm{Fe}$ I & $+0.05 /-0.05$ & $-0.02 /+0.03$ & $-0.03 /+0.03$ \\
$\mathrm{Na}$ I & $+0.03 /-0.04$ & $-0.05 /+0.04$ & $-0.02 /+0.01$ \\
$\mathrm{Mg}$ I & $+0.03 /-0.03$ & $-0.03 /+0.03$ & $-0.02 /+0.02$ \\
$\mathrm{Si} \mathrm{I}$ & $+0.03 /-0.02$ & $-0.01 /+0.02$ & $-0.01 /+0.02$ \\
$\mathrm{Ca}$ I & $+0.05 /-0.04$ & $-0.05 /+0.05$ & $-0.03 /+0.04$ \\
$\mathrm{Ti}$ I & $+0.06 /-0.07$ & $-0.01 /+0.01$ & $-0.03 /+0.02$ \\
$\mathrm{Cr}$ I & $+0.05 /-0.05$ & $-0.01 /+0.02$ & $-0.03 /+0.03$ \\
$\mathrm{Ni}$ I & $+0.05 /-0.04$ & $0.0 /+0.01$ & $-0.04 /+0.03$ \\
$\mathrm{Ba}$ II & $+0.02 /-0.02$ & $+0.04 /-0.05$ & $-0.07 /+0.07$ \\
105495 & & & \\
$\mathrm{Fe}$ I & $+0.01 /+0.03$ & $+0.08 /-0.01$ & $-0.03 /+0.07$ \\
$\mathrm{Na}$ I & $+0.07 /-0.04$ & $-0.05 /+0.04$ & $-0.02 /+0.05$ \\
$\mathrm{Mg}$ I & $0.00 /+0.01$ & $+0.02 /+0.01$ & $-0.03 /+0.04$ \\
$\mathrm{Si}$ I & $-0.05 /+0.06$ & $+0.07 /-0.02$ & $-0.02 /+0.03$ \\
$\mathrm{Ca}$ I & $+0.08 /-0.05$ & $-0.03 /+0.06$ & $-0.04 /+0.07$ \\
$\mathrm{Ti}$ I & $+0.12 /-0.06$ & $+0.03 /+0.02$ & $-0.05 /+0.11$ \\
$\mathrm{Cr}$ I & $+0.08 /+0.02$ & $+0.03 /+0.02$ & $-0.05 /+0.09$ \\
$\mathrm{Ni}$ I & $+0.01 /+0.04$ & $+0.09 /-0.02$ & $-0.04 /+0.08$ \\
$\mathrm{Ba}$ II & $+0.02 /-0.02$ & $+0.05 /-0.05$ & $-0.06 /+0.07$ \\
\hline
\end{tabular}

abundances of elements other than $\mathrm{Fe}$ are expressed as $[\mathrm{X} / \mathrm{Fe}]^{3}$, a total $\sigma_{1}$ should be computed by quadratically adding the rms $\sigma_{1}$ on $[\mathrm{X} / \mathrm{H}]$ and on $[\mathrm{Fe} / \mathrm{H}]$.

The contribution of uncertainties in stellar parameters, $T_{\mathrm{eff}}$, $\log g$, and $\xi$, were estimated by varying each parameter of a given quantity (leaving the other two unchanged) and then adding the three errors. We assumed variations of $\pm 70 \mathrm{~K}$ in $T_{\text {eff }}$ and $\pm 0.10 \mathrm{~km} \mathrm{~s}^{-1}$ in $\xi$, since these changes would introduce significant trends into the relationship of $\mathrm{Fe}$ abundances with $E P$ and observed $E W \mathrm{~s}$. We cannot estimate an uncertainty in $\log g$ in a similar way, since we did not optimize the gravity using the ionization equilibrium. However, errors in $\log g$ are usually of the order of $\sim 0.15-0.25$ dex (e.g., Paper I); thus, we assumed a conservative $\Delta \log g= \pm 0.25$ dex. Table 5 shows the sensitivity of elemental abundance $(\log n(\mathrm{X}))$ to variations in the atmospheric parameters for two cluster members: the TO star 069885 and the clump star 105495 . In the case of Fe we computed $\sigma_{2}$, the quadratic sum of the three errors due to uncertainties in the stellar parameters; this was not done for the other elements, since in the final computation of $[\mathrm{X} / \mathrm{Fe}]$ one should take into account the $\sigma_{2}$ for $[\mathrm{X} / \mathrm{H}]$ and for $[\mathrm{Fe} / \mathrm{H}]$, which could go in opposite directions (see Table 5).

Finally, we wish to give an estimate of the systematic uncertainties in the $\mathrm{Fe}$ abundance scale related to the method of analysis. This can be done for example by analyzing a star with a well-known metallicity, possibly observed with the same instrument and using the same method of analysis. Since we did not collect spectra of stars outside of the clusters included in the program, we performed the analysis for two clump stars in the Hyades observed with SARG at TNG at similar resolution. A detailed description of the analysis of the two Hyades is reported in Paper I; here, we only mention that we did find a $[\mathrm{Fe} / \mathrm{H}]$ in reasonable agreement with the literature estimates, confirming that, up to the metallicity of the Hyades, our method of analysis should not be affected by large systematic errors. To check if the metallicity scale is also correct for a very high metal content, we

${ }^{3}[\mathrm{X} / \mathrm{Fe}]=\log n(\mathrm{X})-\log n(\mathrm{X})_{\odot}-[\mathrm{Fe} / \mathrm{H}]$ for each star, or $[\mathrm{X} / \mathrm{Fe}]=$ $[\mathrm{X} / \mathrm{H}]-[\mathrm{Fe} / \mathrm{H}]$. carried out some tests on $\mu$ Leonis (see Sect. 4.3), a rather luminous giant that is known to have a remarkably oversolar metallicity (e.g., Gratton \& Sneden 1990). We anticipate here that at the metallicity of NGC 6253 our Fe abundance for the coolest star (clump) might be overestimated by $\sim 0.1-0.15$ dex.

\section{Results}

\subsection{Metallicity}

Fe abundances are listed in Table 4, together with their errors, in Cols. 6-8: $\sigma_{1}$, the standard deviation from the mean abundance obtained over the whole set of lines for each star, and $\sigma_{\text {tot }}$, the total uncertainty in which we also consider errors due to stellar parameters $\sigma_{2}\left(\sigma_{\text {tot }}=\sqrt{\sigma_{1}^{2}+\sigma_{2}^{2}}\right)$. Star 023501 was classified as a doubtful member from its radial velocity (see Sect. 2): we found $[\mathrm{Fe} / \mathrm{H}]=+0.29$ for it, in agreement with those of the confirmed members, thus we conclude that this object is a probable cluster member. Stars 069360 and 022182 are instead radial velocity non-members but, as already mentioned, we cannot exclude that they are binary cluster members. The first one has a high Fe abundance (+0.48) similar to those of members; nevertheless, since we do not optimize gravities from the ionization equilibrium and we rely on the photometric values, the derived $[\mathrm{Fe} / \mathrm{H}]$ value might be due only to a coincidence; in other words, if the adopted distance is wrong, one finds a wrong metallicity. On the other hand, if the star would effectively be a binary cluster member, the $[\mathrm{Fe} / \mathrm{H}]$ we found might be the correct one. Star 022182 has a much lower $[\mathrm{Fe} / \mathrm{H}]$ with respect to other stars, that is +0.12 . In any case, we disregard the two non-members in the following. The average metallicity (computed excluding the non-members) with the rms error is also shown in Table 4, $[\mathrm{Fe} / \mathrm{H}]=+0.39 \pm 0.08$.

For the 4 ascertained members we find $[\mathrm{Fe} / \mathrm{H}]=+0.45(\mathrm{TO}$ star), +0.49 (clump), and $+0.32,+0.39$ (SGB/RGB). The metallicity of the clump star 105495 based on $E W$ s could likely be overestimated, due to unresolved blends (the spectrum is very crowded due to the combination of high $\mathrm{Fe}$ content and low temperature); by excluding the clump star, the average metallicity slightly decreases to $[\mathrm{Fe} / \mathrm{H}]=+0.36 \pm 0.07$. A possible offset in the metallicity scale will be discussed in the next sections (4.2 and 4.3). Values shown in parenthesis in Col. 6 of Table 4 are the metallicities found from spectral synthesis for the hottest TO star and for the clump star; the average (+0.34) computed taking into account these values is also reported. Figure 3 shows $[\mathrm{Fe} / \mathrm{H}]$ values as a function of effective temperature for all the stars observed. The average cluster metallicity $\pm \mathrm{rms}$ $([\mathrm{Fe} / \mathrm{H}]=+0.39 \pm 0.08)$ are indicated.

\subsection{Spectral synthesis}

To check Fe abundances derived through the $E W$ analysis, we carried out spectral synthesis for the warmest (069885) and coolest (105495) cluster stars, which are also those having the highest $[\mathrm{Fe} / \mathrm{H}]$. The spectral synthesis was performed in a spectral interval of $\sim \pm 10 \AA$ around the Li I $6707.8 \AA$ line. As for the $E W$ analysis, we used MOOG and Kurucz atmospheres; an earlier version of MOOG (2000) was, however, employed, with a line list optimized to fit the solar spectrum obtained with UVES; the Fe abundances retrieved from the synthesis are therefore differential with respect to the Sun. We used the classical Unsöld (1955) approximation for the damping coefficients, since the spectral range investigated with the synthesis does not include strong lines. 


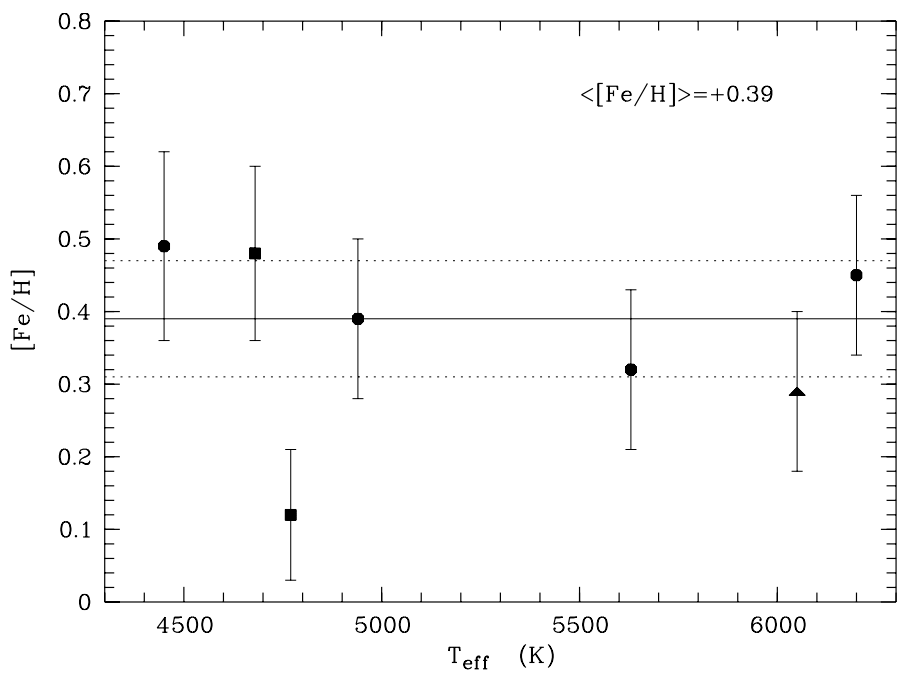

Fig. 3. Fe abundances as a function of $T_{\text {eff }}$ for stars in NGC 6253. Symbols for members, the possible member and non-members are the same as in Fig. 1. The solid line represents the average Fe content (including all the stars but the non-members), while the dashed ones represent the rms.

Synthetic spectra were computed adopting stellar parameters derived by the $E W$ analysis and listed in Table 4 . For each star, we computed a synthetic spectrum with the metallicities determined through $E W \mathrm{~s}$ and then others until the best fit of the observed spectrum was obtained. Figure 4 shows the spectral synthesis for two stars (105495 and 069885) in the wavelength range $6700-6718 \AA$. In the upper panel, we plot the comparison between the observed spectrum of the clump star and two synthetic spectra with $[\mathrm{Fe} / \mathrm{H}]=+0.35$ and +0.49 : as is evident, the lines of $\mathrm{Fe} \mathrm{I}$ in the synthetic spectrum with metallicity +0.49 are in the majority of cases deeper than the observed ones, while the synthesis with $[\mathrm{Fe} / \mathrm{H}]=+0.35$ provides a better fit (although far from perfect). This also happens for the TO star (lower panel) for which we computed two synthesis with $[\mathrm{Fe} / \mathrm{H}]=+0.35$ and +0.45 . Therefore, in both cases the metallicity obtained from spectral synthesis is $\sim 0.1$ dex below that determined using $E W$ s. A higher metallicity from $E W$ analysis with respect to synthesis can easily be explained for the clump star, whose spectrum might be affected by blending; on the other hand, the discrepancy found for the TO star is rather surprising, and we do not really have an explanation for this.

As mentioned in Sect. 4.1, by assuming $[\mathrm{Fe} / \mathrm{H}]=+0.35$ for the TO and clump stars, the average $[\mathrm{Fe} / \mathrm{H}]$ of the cluster would decrease down to +0.34 . However, we stress that errors in the determination of the continuum in the observed spectra also affect the comparison with synthetic spectra and not only $E W$ measurements. Finally, note that in the wavelength region shown in Fig. 4 there are observed spectral features not reproduced by the synthesis: these are lines of elements other than Fe, which in this case are not important for the determination of the metallicity (e.g., the Ti I line at $6706.3 \AA$ and the Li I line at $6707.8 \AA$ ) and for which, therefore, we did not optimize atomic parameters and abundances.

\subsection{Comparison with $\mu$ Leo}

Since the analysis through EWs and the spectral synthesis yield slightly different results, we carried out a further test to check our metallicity scale: namely, we analyzed the metal-rich giant star $\mu$ Leonis. The values of $[\mathrm{Fe} / \mathrm{H}]$ estimated in the literature are all around +0.3-0.4 dex (e.g., Gratton \& Sneden 1990, $[\mathrm{Fe} / \mathrm{H}]=+0.34$; Fulbright et al. 2006, $[\mathrm{Fe} / \mathrm{H}]=+0.32$; Gratton et al. 2006, $[\mathrm{Fe} / \mathrm{H}]=+0.38$ ), therefore similar to that of NGC 6253. We analyzed a spectrum of $\mu$ Leo observed with FEROS on the $2.2 \mathrm{~m}$ telescope at La Silla Observatory with a resolution similar to that of our sample stars. By using the same line list as for NGC 6253, we obtained $[\mathrm{Fe} / \mathrm{H}]=+0.51$ for $\mu$ Leo, and $T_{\text {eff }}=4400 \mathrm{~K}, \log g=2.3$, and $\xi=1.2 \mathrm{~km} \mathrm{~s}^{-1}$; whereas the atmospheric parameters are in good agreement with the determinations of other authors, the Fe abundance is higher than previously found. A metallicity more similar to those quoted by other authors is obtained by us with the spectral synthesis, i.e., $[\mathrm{Fe} / \mathrm{H}]=+0.38$; since by spectral synthesis we find $[\mathrm{Fe} / \mathrm{H}]=+0.35$ for 105495 , which has atmospheric parameters similar to those of $\mu$ Leo, our results suggest that the clump star in NGC 6253 and $\mu$ Leo should actually have similar metallicities, but a scale offset is present between the synthesis and $E W$ analysis. Figure 5 shows a comparison between the spectra of $\mu$ Leo and 105495 in the wavelength region around the Li I doublet at $6708 \AA$, where several Fe I features are present: as is clearly visible, the spectral lines of the two stars are similar, suggesting that the metallicities are nearly the same. In particular, $\mu$ Leo is slightly colder than 105495, and indeed its metal lines are slightly stronger. In any case, the problem of the determination of a zero-point for the $[\mathrm{Fe} / \mathrm{H}]$ scale remains.

To further check the metallicity scale and the origin of the discrepancy, we carried out different tests on $\mu$ Leo. We considered the line list for Fe I adopted by Fulbright et al. (2006): more in detail, using the 30 lines in common with our list we carried out an $E W$ analysis adopting our atomic parameters and their $E W$ s. The analysis by Fulbright et al. (2006) is differential with respect to the Sun, for which they derive $\log n(\mathrm{Fe}) \odot=7.45$. With their measurements and our atomic parameters we find $[\mathrm{Fe} / \mathrm{H}]=+0.40$, i.e., 0.08 dex larger than that of Fulbright et al. (2006; +0.32$)$. Note that we obtain $T_{\text {eff }}=4550 \mathrm{~K}$, higher than the value we previously found, but in agreement with Fulbright et al. (2006). If we adopt the 30 lines in common with Fulbright et al. (2006), but using our $E W \mathrm{~s}$, we find a metallicity $[\mathrm{Fe} / \mathrm{H}]=$ +0.49 , with $T_{\text {eff }}=4450 \mathrm{~K}$. Finally, we also repeated the latter analysis for the clump star 105495 in NGC 6253, i.e., with the lines in common with Fulbright et al. (2006 - and obviously our $E W \mathrm{~s}$ ), and we obtained $[\mathrm{Fe} / \mathrm{H}]=+0.44$. Part of the discrepancies with the previous analysis can be due to $E W$ measurements (program/method and continuum tracing) and the adopted code, but in any case we obtain a systematically higher metallicity.

Given the disagreement found with the literature results for $\mu$ Leo, and between the spectral synthesis and $E W$ analysis for stars in NGC 6253, we conclude that an offset in the abundance of the clump star is present (probably due to a combination of low $T_{\text {eff }}$ and high $[\mathrm{Fe} / \mathrm{H}]$ that results in very strong and blended features); whereas we are not able to precisely quantify the offset, we note that it is in the range $\sim+0.10-0.15 \mathrm{dex}$; for the other stars we cannot estimate a possible abundance shift through a similar comparison with a well-known star, since we do not know a very metal-rich subgiant to be used as a reference object. In summary, we will adopt the $[\mathrm{Fe} / \mathrm{H}]$ values derived through $E W$ analysis for all the stars, but with the caveat that those of the clump and of the hottest TO stars are probably overestimated, while the metallicity found for the remaining stars is likely to be correct.

As far as other elements are concerned, it has not been possible to carry out a direct comparison with $\mu$ Leo. Although a chemical analysis of this star has recently been performed by 

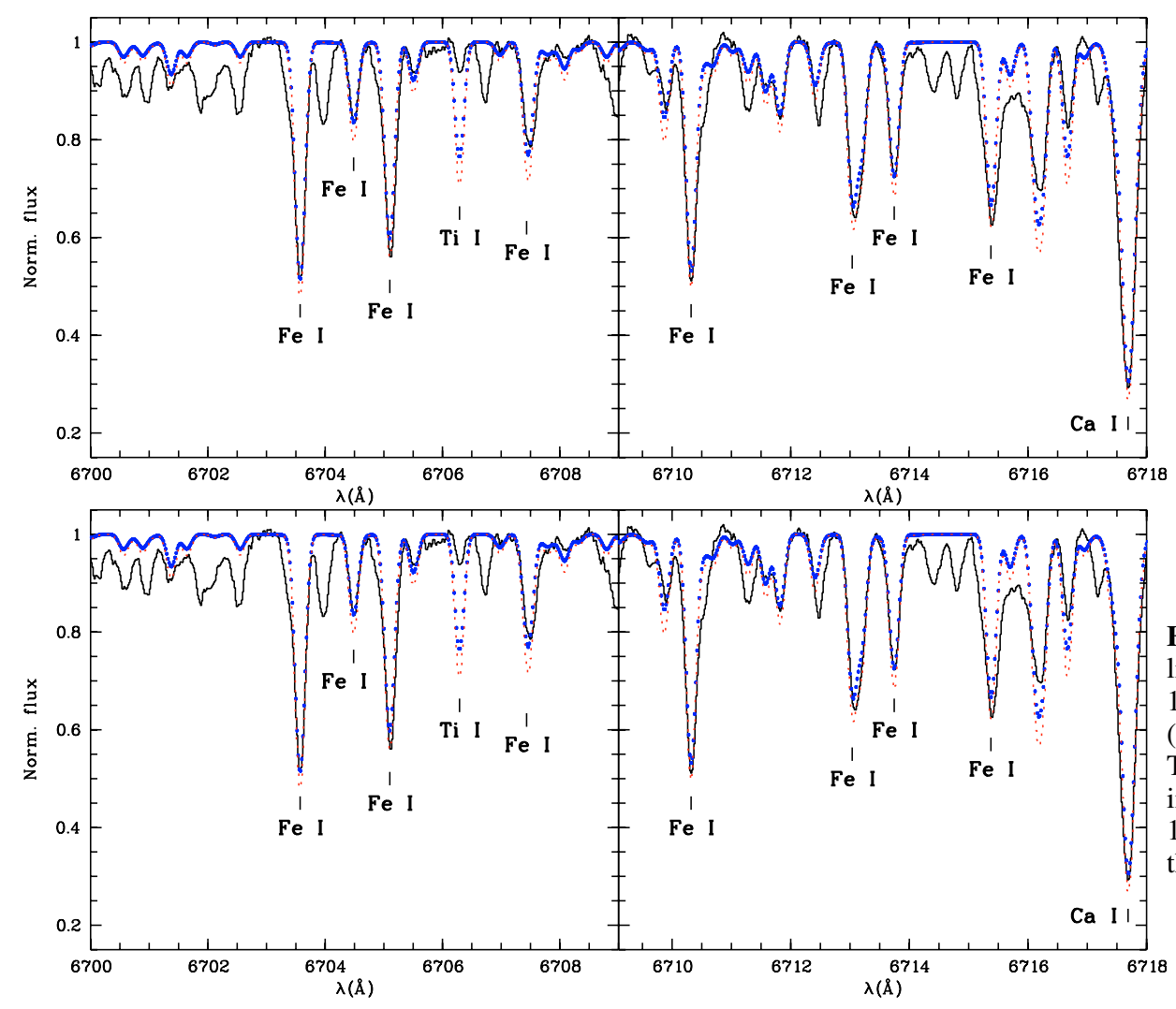

Fig. 4. Comparison between observed (solid line) and synthetic spectra for the clump star 105495 (upper panel) and the TO star 069885 (lower panel) in the Li I region (6707.8 $\AA$ ). The (red) thin dots are spectra computed adopting the spectroscopic metallicities $(+0.49$ for 105495 and +0.45 for 069885 ), while the (blue) thick dots are spectra with $[\mathrm{Fe} / \mathrm{H}]=+0.35$.

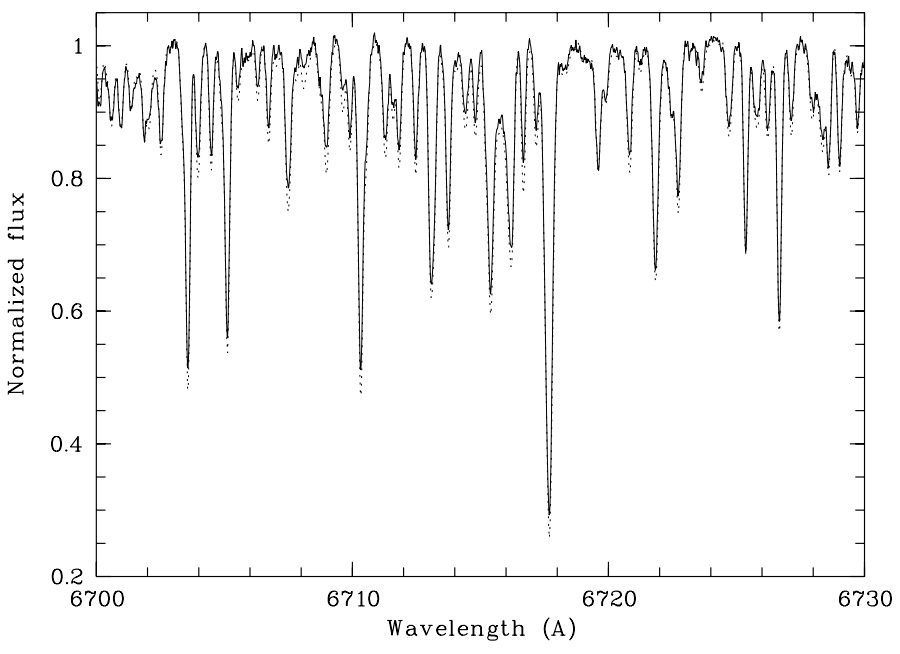

Fig. 5. Comparison between the spectra of the clump star 105495 in NGC 6253 (solid line) and of $\mu$ Leo (dashed line) in the region of the Li I feature (6707.8 ̊).

Fulbright et al. (2007), their line lists and ours have only a small number of lines (if any) in common for each element; moreover, their $E W \mathrm{~s}$ were not published. By comparing the abundances for $\alpha$-elements ( $\mathrm{Ca}$ and $\mathrm{Si}$ in particular) obtained using the few lines in common with Fulbright et al. (2007) and our EWs, we obtain $[\mathrm{X} / \mathrm{H}]$ values similar to theirs, suggesting that the scale offset is likely to affect only Fe.

\subsection{Abundances of other elements}

Besides $\mathrm{Fe}$, we derived the abundances of the light isotope $\mathrm{Na}$, the $\alpha$-elements $\mathrm{Mg}, \mathrm{Si}, \mathrm{Ca}, \mathrm{Ti}$, the Fe-peak elements $\mathrm{Cr}$ and $\mathrm{Ni}$, and the s-process element $\mathrm{Ba} .[\mathrm{X} / \mathrm{H}]$ values for $\mathrm{Si}, \mathrm{Ca}, \mathrm{Ti}, \mathrm{Cr}$, $\mathrm{Ni}$, and $\mathrm{Ba}$ are listed in Table 6 , together with the errors due to uncertainties in $E W \mathrm{~s}\left(\sigma_{1}\right)$. The $\log n(\mathrm{X})$ values in the Sun found by us (with the exception of $\mathrm{Ba}$ ), are also shown (last column). $[\mathrm{X} / \mathrm{Fe}]$ ratios are instead shown in Table 7 , with errors computed by quadratically adding the $\mathrm{rms} \sigma_{1}$ for $[\mathrm{Fe} / \mathrm{H}]$ and for $[\mathrm{X} / \mathrm{H}]$. We notice that for $\mathrm{Ba}$ and $\mathrm{Si}$ the measurement is based on a small sample of lines (3 and 4-6 features, respectively). For the other species $(\mathrm{Ca}, \mathrm{Ti}, \mathrm{Cr}$, and $\mathrm{Ni})$, we carried out the analysis using a large set of lines, and we performed $1 \sigma$ clipping. In the last three columns of Table 7 we report two average values $( \pm \mathrm{rms})$ of the $[\mathrm{X} / \mathrm{Fe}]$ abundances for the cluster. The means are computed including all the five stars (Average1), or excluding the clump star (Average2). In general the two averages are consistent with each other within the errors; the scatter is smaller if only the confirmed members are considered.

As discussed in further detail by Bragaglia et al. (in preparation), we find a rather large error $( \pm 0.14)$ on the solar $\log n(\mathrm{Mg})$, and this might depend on uncertainties on the $\log g f \mathrm{~s}$ and on the fact that the analysis of this species is based on a very small number of lines. The analysis of Na usually is based on a set of seven lines, but in the case of NGC 6253 not all these features are measurable and in addition the few lines used give discrepant results. For this reason, the ratios of $\mathrm{Na}$ and $\mathrm{Mg}$ to $\mathrm{Fe}$ in stars of NGC 6253 have been computed from a line-to-line comparison, instead of comparing the average abundance of a star to the solar one. The abundances of the two elements are presented separately in Table 8 , where we report $[\mathrm{X} / \mathrm{H}]$ and $[\mathrm{X} / \mathrm{Fe}]$ for each line in common between the lists adopted for the Sun and for the cluster stars.

We mention that for $\mathrm{Mg}$ only two lines were used, and, as a consequence, the results shown in Table 8, which indicate a $\mathrm{Mg}$ enhancement, should be taken with caution and would require a dedicated study that goes beyond the primary goal of the 
Table 6. Elemental ratios ([X/H]) for stars in NGC 6253: $\mathrm{Si}, \mathrm{Ca}, \mathrm{Ti}, \mathrm{Cr}, \mathrm{Ni}$, and $\mathrm{Ba}$. Errors are the rms $\sigma_{1}-$ due to $E W$ uncertainties - on [X/H]. The solar abundances we found are also shown $\left(\log n(\mathrm{X})_{\odot}\right)$.

\begin{tabular}{ccccccc}
\hline \hline Element & 069885 & 023501 & 023498 & 024707 & 105495 & Sun $\left(\log n(\mathrm{X})_{\odot}\right)$ \\
\hline Si I & $+0.44 \pm 0.12$ & $+0.21 \pm 0.04$ & $+0.35 \pm 0.98$ & $+0.40 \pm 0.17$ & $+0.35 \pm 0.17$ & $7.60 \pm 0.03$ \\
$\mathrm{Ca}$ I & $+0.50 \pm 0.08$ & $+0.32 \pm 0.12$ & $+0.32 \pm 0.10$ & $+0.35 \pm 0.12$ & $+0.25 \pm 0.10$ & $6.35 \pm 0.04$ \\
$\mathrm{Ti} \mathrm{I}$ & $+0.44 \pm 0.10$ & $+0.30 \pm 0.10$ & $+0.21 \pm 0.08$ & $+0.59 \pm 0.11$ & $+0.34 \pm 0.08$ & $4.93 \pm 0.03$ \\
$\mathrm{Cr}$ I & $+0.45 \pm 0.09$ & $+0.29 \pm 0.07$ & $+0.36 \pm 0.11$ & $+0.49 \pm 0.17$ & $+0.33 \pm 0.15$ & $5.66 \pm 0.03$ \\
$\mathrm{Ni}$ I & $+0.44 \pm 0.09$ & $+0.34 \pm 0.09$ & $+0.38 \pm 0.08$ & $+0.50 \pm 0.09$ & $+0.67 \pm 0.11$ & $6.26 \pm 0.02$ \\
$\mathrm{Ba}$ II & $+0.75 \pm 0.13$ & $+0.40 \pm 0.15$ & $+0.63 \pm 0.14$ & $+0.57 \pm 0.16$ & $+0.73 \pm 0.04$ & $2.13 *$ \\
\hline
\end{tabular}

* The solar Ba abundance is from Anders \& Grevesse (1989).

Table 7. $[\mathrm{X} / \mathrm{Fe}]$ abundances and averages. Errors are the quadratic sum of $\sigma_{1}$ on $[\mathrm{X} / \mathrm{H}]$ and on $[\mathrm{X} / \mathrm{Fe}]$.

\begin{tabular}{cccccccc}
\hline \hline Element & 069885 & 023501 & 023498 & 024707 & 105495 & Average $1 \pm$ rms & Average2 \pm rms \\
\hline Si I & $-0.01 \pm 0.15$ & $-0.08 \pm 0.10$ & $+0.03 \pm 0.13$ & $+0.01 \pm 0.19$ & $+0.14 \pm 0.20$ & $+0.02 \pm 0.08$ & $-0.01 \pm 0.05$ \\
$\mathrm{Ca}$ I & $+0.05 \pm 0.12$ & $+0.03 \pm 0.15$ & $0.00 \pm 0.13$ & $-0.04 \pm 0.15$ & $-0.24 \pm 0.14$ & $-0.04 \pm 0.12$ & $+0.01 \pm 0.04$ \\
$\mathrm{Ti}$ I & $-0.01 \pm 0.13$ & $+0.01 \pm 0.13$ & $-0.11 \pm 0.14$ & $+0.20 \pm 0.14$ & $-0.15 \pm 0.13$ & $-0.01 \pm 0.14$ & $+0.02 \pm 0.13$ \\
$\mathrm{Cr}$ I & $+0.01 \pm 0.13$ & $0.00 \pm 0.11$ & $+0.04 \pm 0.24$ & $+0.10 \pm 0.19$ & $-0.16 \pm 0.18$ & $-0.02 \pm 0.10$ & $+0.04 \pm 0.05$ \\
$\mathrm{Ni}$ I & $-0.01 \pm 0.13$ & $+0.05 \pm 0.15$ & $+0.06 \pm 0.12$ & $+0.11 \pm 0.13$ & $+0.18 \pm 0.15$ & $0.08 \pm 0.07$ & $+0.05 \pm 0.05$ \\
$\mathrm{Ba}$ II & $+0.30 \pm 0.16$ & $+0.11 \pm 0.17$ & $+0.31 \pm 0.17$ & $+0.18 \pm 0.18$ & $+0.24 \pm 0.11$ & $+0.23 \pm 0.08$ & $+0.23 \pm 0.10$ \\
\hline
\end{tabular}

Averages: 1: computed including all the 5 stars; 2: computed excluding the clump star 105495.

Table 8. Abundances of Mg and Na (LTE and non-LTE) computed for each line adopted.

\begin{tabular}{|c|c|c|c|c|c|c|c|c|c|c|c|}
\hline \multirow{2}{*}{ Wavelength } & \multirow[b]{2}{*}{$\log n(\mathrm{X})_{\odot}$} & \multicolumn{2}{|r|}{069885} & \multicolumn{2}{|r|}{023501} & \multicolumn{2}{|r|}{023498} & \multicolumn{2}{|r|}{024707} & \multicolumn{2}{|r|}{105495} \\
\hline & & {$[\mathrm{X} / \mathrm{H}]$} & {$[\mathrm{X} / \mathrm{Fe}]$} & {$[\mathrm{X} / \mathrm{H}]$} & $\overline{[\mathrm{X} / \mathrm{Fe}]}$ & $\begin{array}{l}{[\mathrm{X} / \mathrm{H}]} \\
\mathrm{I}\end{array}$ & {$[\mathrm{X} / \mathrm{Fe}]$} & {$[\mathrm{X} / \mathrm{H}]$} & {$[\mathrm{X} / \mathrm{Fe}]$} & {$[\mathrm{X} / \mathrm{H}]$} & {$[\mathrm{X} / \mathrm{Fe}]$} \\
\hline 6318.71 & 7.54 & +0.42 & -0.03 & +0.71 & +0.42 & +0.75 & +0.43 & +0.72 & +0.33 & +0.86 & +0.37 \\
\hline 6319.24 & 7.47 & +0.65 & +0.20 & - & - & +0.41 & +0.09 & +0.65 & +0.26 & +0.94 & +0.45 \\
\hline Average & & & $+0.09 \pm 0.16$ & & +0.42 & & $+0.26 \pm 0.24$ & & $+0.30 \pm 0.05$ & & $+0.41 \pm 0.06$ \\
\hline & & & & & & & & & & & \\
\hline 5688.22 & 6.20 & +0.69 & +0.24 & +0.42 & +0.13 & +0.77 & +0.45 & +0.40 & +0.01 & +0.44 & -0.05 \\
\hline 6154.23 & 6.29 & +0.52 & +0.07 & +0.51 & +0.22 & +0.61 & +0.29 & +0.75 & +0.36 & +0.88 & +0.39 \\
\hline 6160.75 & 6.30 & +0.60 & +0.15 & +0.21 & -0.08 & +0.51 & +0.19 & +0.69 & +0.30 & +0.77 & +0.28 \\
\hline Average & & & $+0.15 \pm 0.09$ & & $+0.09 \pm 0.15$ & & $+0.31 \pm 0.13$ & & $+0.22 \pm 0.19$ & & $+0.21 \pm 0.23$ \\
\hline Wavelength & & & {$[\mathrm{Na} / \mathrm{Fe}]_{\text {non-LTE }}$} & & {$[\mathrm{Na} / \mathrm{Fe}]_{\text {non-LTE }}$} & & {$[\mathrm{Na} / \mathrm{Fe}]_{\text {non-LTE }}$} & & {$[\mathrm{Na} / \mathrm{Fe}]_{\text {non-LTE }}$} & & {$[\mathrm{Na} / \mathrm{Fe}]_{\text {non-LTE }}$} \\
\hline 5688.22 & & & +0.12 & & +0.00 & & +0.32 & & -0.14 & & -0.20 \\
\hline 6154.23 & & & -0.05 & & +0.10 & & +0.17 & & +0.27 & & +0.24 \\
\hline 6160.75 & & & +0.03 & & -0.20 & & +0.02 & & +0.21 & & +0.13 \\
\hline Average & & & $+0.03 \pm 0.09$ & & $-0.03 \pm 0.15$ & & $+0.17 \pm 0.15$ & & $+0.11 \pm 0.22$ & & $+0.06 \pm 0.23$ \\
\hline
\end{tabular}

present paper. We used three spectral features for the determination of $\mathrm{Na}$ abundance, but the two lines at 6154-6160 $\AA$ are likely to be more reliable than the $5688 \AA$ line, which is rather strong and might deserve more detailed damping computations. $\mathrm{Na}$ abundances derived with MOOG are based on the assumption of LTE. As is well known, this assumption may introduce systematic errors in the computation of the abundance; whereas for most of the elements it has been ascertained that non-LTE corrections are negligible, in the case of $\mathrm{Na}$ they might be important. The problem is that non-LTE effects affect stars in diverse evolutionary phases (main sequence, TO, RGB, clump) at different levels since they are strongly dependent on the temperature and surface gravity. Also, discrepant results have been obtained from different authors in the computation of non-LTE corrections. For example, Gratton et al. (1999) find moderate negative corrections of the order of $\sim 0.05-0.1$ dex for giant stars, while Mashonkina et al. (2000) estimate larger corrections, of the order of $\sim 0.15$ dex. In Table 8 we show $[\mathrm{Na} / \mathrm{Fe}]$ values derived with MOOG and corrected adopting the tabulations by Mashonkina et al. (2000). Considering LTE abundances, Na seems to be enhanced with respect to the solar value, in agreement with other findings for open clusters (Friel et al. 2003; Yong et al. 2005;
Bragaglia et al. 2006), but a certain amount of scatter is present in the abundances from the various lines and also among the various stars. On the other hand, with the non-LTE corrections by Mashonkina et al. (2000) the $[\mathrm{Na} / \mathrm{Fe}]$ values end up being lower by $\sim 0.10-0.15$ dex depending on the line considered and on the stellar parameters. In this case, the average $\mathrm{Na}$ abundance of the cluster would be nearly or slightly above solar; therefore, we suggest that the $\mathrm{Na}$ abundance enhancement claimed for open clusters based on giant stars might be in part related to nonLTE effects (see also Randich et al. 2006). On the other hand, the $[\mathrm{Na} / \mathrm{Fe}]$ abundance ratios of field dwarfs do not appear to be enhanced (e.g., Soubiran \& Girard 2005); this issue deserves further investigation, which is beyond the goals of this paper.

In Fig. 6 we show the element abundances of Table 7 as a function of $[\mathrm{Fe} / \mathrm{H}]$; also in this case the mean abundance $\pm \mathrm{rms}$ are represented. We adopted the value Average1 shown Table 7, i.e., computed including all the 5 stars. All the elements, apart from $\mathrm{Ba}$, have average solar abundances; $\mathrm{Ba}$ is enhanced, as already found for other clusters, e.g., by our group (Bragaglia et al., in preparation) or by Bragaglia et al. (2006); however, abundance for this element has been found to vary significantly between clusters (e.g., Gratton et al. 2004). 

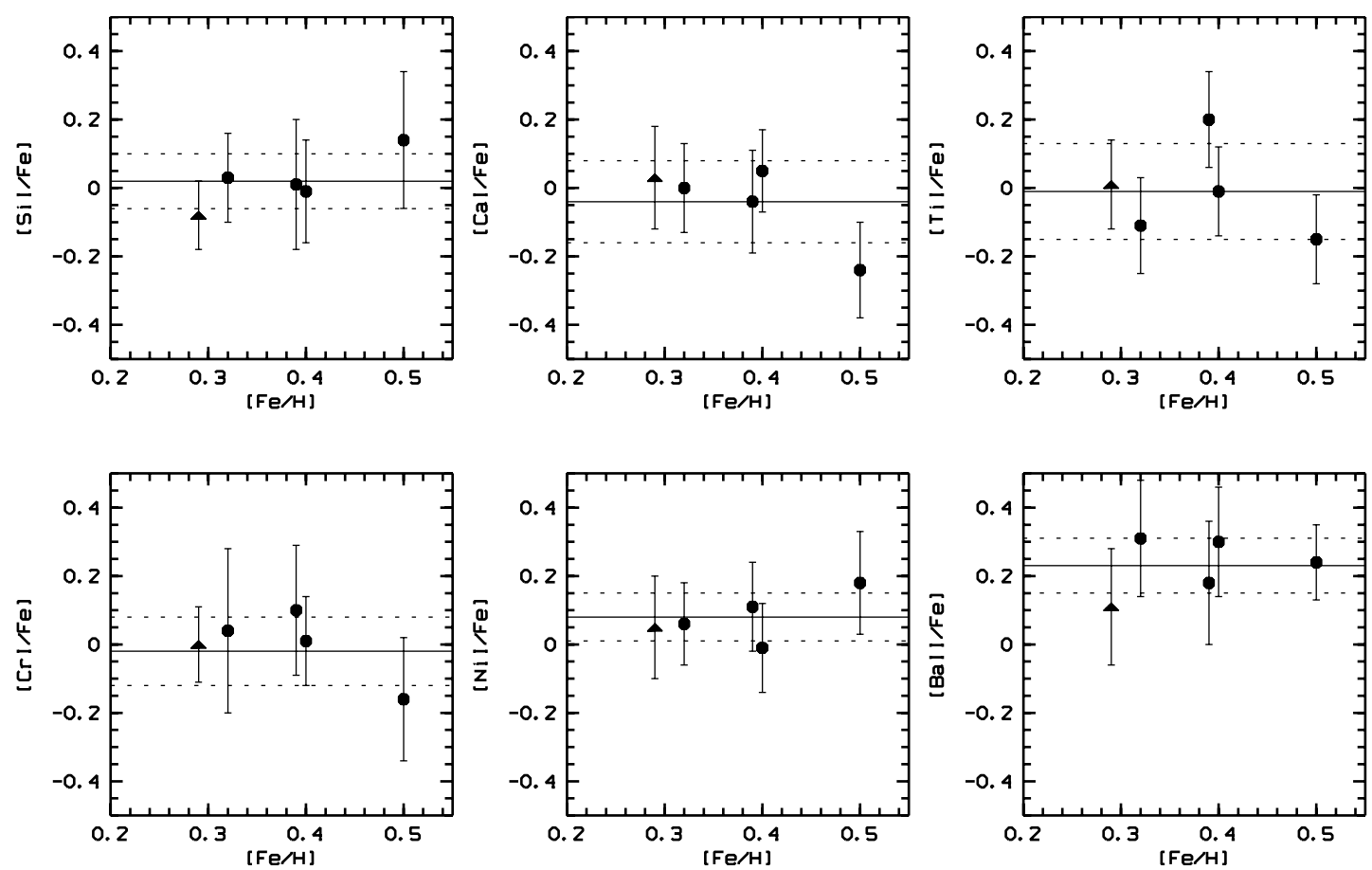

Fig. 6. $[\mathrm{X} / \mathrm{Fe}]$ abundances of the various elements analyzed as a function of $[\mathrm{Fe} / \mathrm{H}]$. The solid and dotted lines are the averages \pm rms (computed including all the 5 stars plotted; Average1 in Table 7); the triangle represents the possible member.
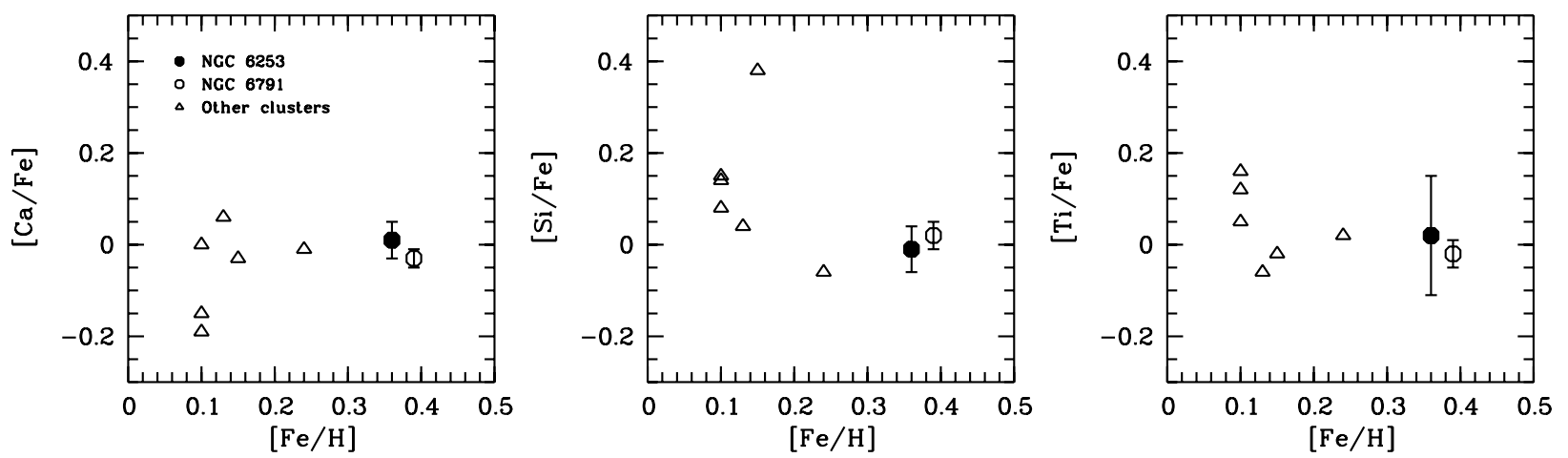

Fig. 7. $\alpha$-element abundances vs. [Fe/H]: comparison between NGC 6253 (filled circle), NGC 6791 (open circle, Carraro et al. 2006), and other open clusters with oversolar $[\mathrm{Fe} / \mathrm{H}]$ (open triangles). For NGC 6253, at variance with previous figures, we adopt here and in Figs. 8 and 9 the mean computed excluding the clump star (called Average2 in Table 7).

\section{Discussion}

As is mentioned in the introduction, the metallicity of NGC 6253 is unusual for disk stars, which normally have abundances close to solar. The only other cluster for which a very high Fe content has been reported is the 9 Gyr old NGC 6791. Carraro et al. (2006) and Origlia et al. (2006) found $[\mathrm{Fe} / \mathrm{H}]=+0.39$ and +0.35 , respectively, and solar $\alpha$-element abundances. NGC 6791 is a peculiar cluster, since it is very massive and has a very eccentric orbit; therefore, it is different from NGC 6253 in several aspects. In particular, NGC 6253 is much younger than NGC 6791 and much less massive; the orbit of NGC 6253 has not been studied yet, but it could be interesting to have information on it. Given its position towards the Galactic center, NGC 6253 could have been born either towards the bulge, where the metallicity is high, or in a region of the disk where a particular metal enrichment occurred. To get insights on the origin of this cluster, in Fig. 7 we show a comparison for $\alpha$-element abundances ( $\mathrm{Si}, \mathrm{Ca}$, Ti) vs. $[\mathrm{Fe} / \mathrm{H}]$ in NGC 6253, NGC 6791, and other open clusters with $[\mathrm{Fe} / \mathrm{H}] \gtrsim+0.10$; the clusters are the Hyades, NGC 5822,
IC 4651, IC 4725, and NGC 6705 (references can be found in Friel 2006 and Randich 2007). We plotted these three elements since their analysis is based on a rather large sample of lines with respect to the other species, and they are all explosive nucleosynthesis $\alpha$-elements, i.e., they originate from type II Supernova events. Note that for NGC 6253 we consider here (and in Figs. 8 and 9) the values labeled as Average 2 in Table 7, excluding the clump star; similarly, for Fe we adopted the value +0.36 , again excluding the clump star. The figure shows that the average $\alpha$ element abundances of NGC 6791 and NGC 6253 are identical or in very good agreement. $\alpha$-element abundances in open clusters with oversolar Fe content are in general close to solar (with the exception of a Si enhancement in IC 4725, Luck et al. 1994).

Figure 8 shows a comparison between NGC 6253 and disk dwarfs observed by Mishenina et al. (2004; thin and thick disk) and Bensby et al. (2005; thick disk). Also in this case the $\alpha$ elements $\mathrm{Si}, \mathrm{Ca}$ and $\mathrm{Ti}$ were considered; the figure shows the range in metallicity $[\mathrm{Fe} / \mathrm{H}] \sim-0.1 \div+0.4$. The average abundances of evolved stars in NGC 6253 match the general trend 

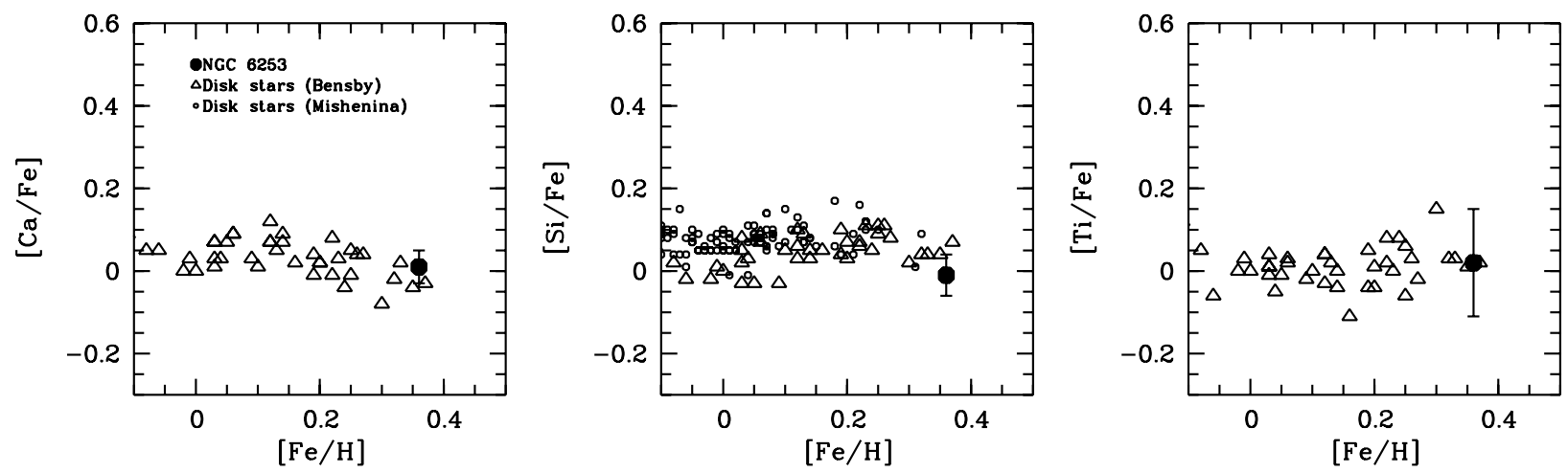

Fig. 8. $\alpha$-element abundances vs. [Fe/H]: comparison between NGC 6253 (filled circle) and disk stars (open triangles, Bensby et al. $2005-$ thick disk; open circles, Mishenina et al. 2004 - thick and thin disk). In the latter study, $\mathrm{Ca}$ and Ti were not investigated.
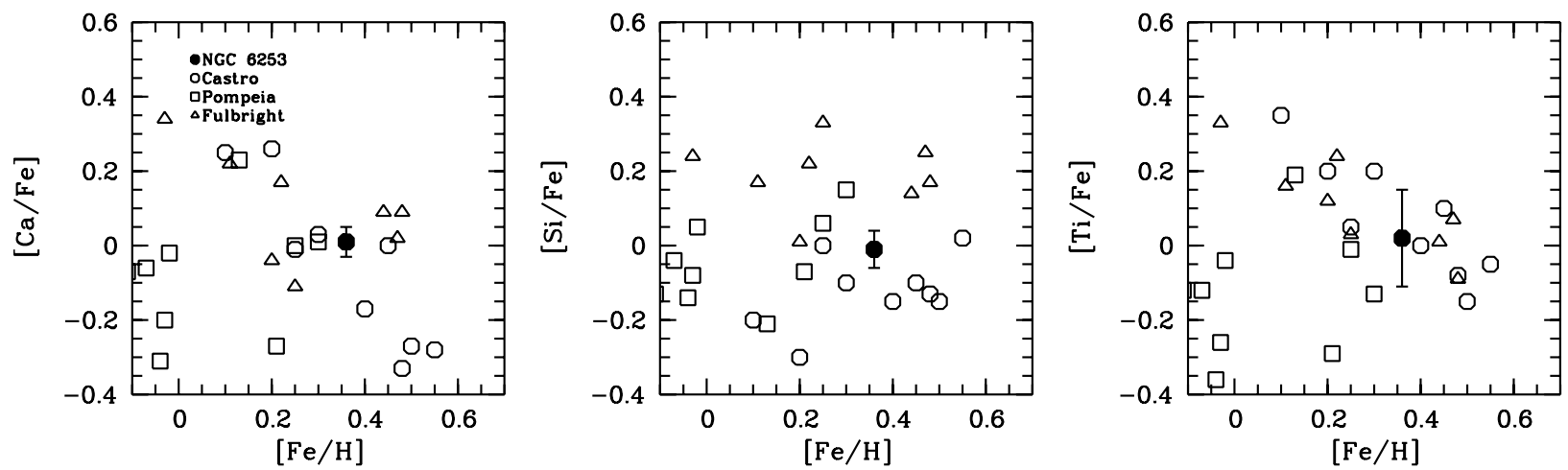

Fig. 9. $\alpha$-element abundances vs. [Fe/H]: comparison between NGC 6253 (filled circle), bulge stars (open triangles, Fulbright et al. 2007), and bulge-like field stars observed by Castro et al. (1997, open circles) and Pompeia et al. (2003, open squares).

observed for thin and thick disk dwarfs very well. Finally, in Fig. 9 we plot a comparison between NGC 6253 and the results for bulge giant stars recently analyzed by Fulbright et al. (2007) and bulge-like field stars by Castro et al. (1997) and Pompeia et al. (2003). In the latter works samples of nearby dwarfs with kinematics and metallicity characteristics of a probable inner disk or bulge origin have been investigated. The bulge and bulgelike field star samples cover a $[\mathrm{Fe} / \mathrm{H}]$ range $\sim-1.20 \div+0.60$, but we show abundances only for $[\mathrm{Fe} / \mathrm{H}]$ larger than $\sim-0.1$. Bulge and bulge-like stars are characterized by $\alpha$-enhancement at very low metallicities (not visible in the figure) with a decrease towards solar and oversolar metallicities, as shown in the plot; note, however, the much larger dispersion with respect to disk stars reported in Fig. 8: indeed there are stars showing enhanced $\alpha$-element abundances at solar/oversolar metallicities also.

The comparisons between NGC 6253 and field stars suggest that the abundance of the cluster is in good agreement with the trend observed in the disk (Fig. 8). From this evidence, we can speculate that NGC 6253 was born in the Galactic disk, in a region where a larger than normal Fe enrichment occurred; on the other hand, we do not observe an enhancement of $\alpha$-elements with respect to $\mathrm{Fe}$. Under the assumption that the cluster formed in the disk, we can use it for the determination of the radial metallicity gradient. In Fig. 10 we show $[\mathrm{Fe} / \mathrm{H}]$ as a function of the Galactocentric radius for NGC 6253 and the other samples included in our program (Paper I and Bragaglia et al., in preparation), compared to other samples analyzed with high resolution spectroscopy (references for all the clusters investigated at high resolution can be found in Paper I). In the figure, we also show the low-resolution sample by Friel et al. (2002; but note that we excluded the clusters in common with high resolution

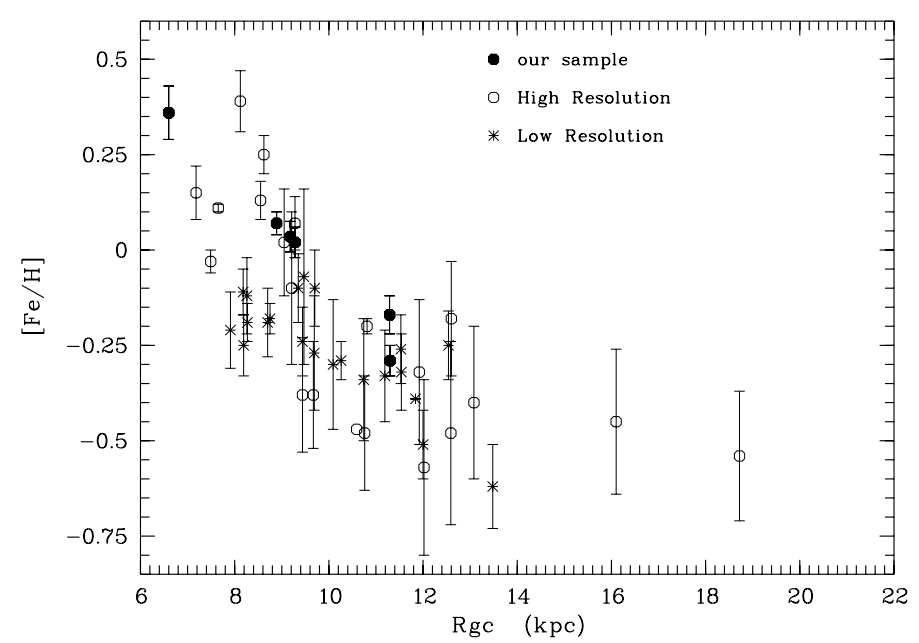

Fig. 10. Radial gradient ([Fe/H] vs. Galactocentric distance) for open clusters. The results for clusters in our sample analyzed so far (filled circles; this paper, Paper I and Bragaglia et al., in preparation) are compared to other clusters analyzed with high-resolution spectroscopy (open circles, see Paper I for references) and low-resolution spectroscopy (asterisks, Friel et al. 2002).

studies). For consistency, we adopt for all the clusters $R_{\mathrm{gc}}$ from Friel (1995) and Friel et al. (2002).

NGC 6791 (the cluster with the highest $[\mathrm{Fe} / \mathrm{H}]$ ) lies above the average trend for open clusters, confirming that it might have had an origin and evolutionary history very different from those of other clusters (Carraro et al. 2006). Also the very metal-rich NGC 6253 (the other cluster with $[\mathrm{Fe} / \mathrm{H}]>+0.3$ ) and Praesepe 
$([\mathrm{Fe} / \mathrm{H}]=+0.25$; Pace et al., in preparation $)$ might lie above the mean trend. In other words, the inclusion of NGC 6253 in the $[\mathrm{Fe} / \mathrm{H}]$ vs. $R_{\mathrm{gc}}$ distribution makes the negative slope of the gradient steeper. However, we note that all the clusters with $R_{\mathrm{gc}}$ lower than that of the Sun $-\sim 8.5 \mathrm{kpc}$ - have higher than solar metallicities, with the exception of $\mathrm{Cr} 261$, which has $[\mathrm{Fe} / \mathrm{H}]=-0.03$ (Carretta et al. 2005; De Silva et al. 2006). This is true for high resolution spectroscopy results, whereas Friel et al. (2002), from low resolution data, quote metallicities from solar down to -0.25 dex for clusters with $R_{\mathrm{gc}}$ between 8 and $8.5 \mathrm{kpc}$. Indeed, the metallicities by Friel et al. (2002) from low resolution data are - for all the clusters and at all $R_{\mathrm{gc}} \mathrm{s}$ - lower than those obtained from high resolution analysis. Considering only $[\mathrm{Fe} / \mathrm{H}]$ values from high resolution and clusters with $R_{\mathrm{gc}}<8.5 \mathrm{kpc}$, we note that $\mathrm{Cr} 261$ might represent an exception for its relatively low Fe content, rather than NGC 6253 and other high metallicity clusters. To our knowledge, no investigations of the orbit of $\mathrm{Cr}$ 261 are present in the literature, but it would be very interesting to have such information, to understand if this cluster might have formed at a larger Galactocentric distance than its present position.

We tentatively conclude that the problems/questions raised by the very high metallicity of NGC 6791 should not really concern NGC 6253, which is rather young and is located towards the Galactic center and very close to it (at variance with NGC 6791, which is is much older and is not located in the Galactic center direction). Finally, we would like to remark that, as mentioned in Sect. 1, Twarog et al. (2003) suggested the possibility of an $\alpha$ element enhancement in NGC 6253. We find instead solar-scaled abundances for these elements, implying that stellar evolutionary models at these metallicities also need to be improved.

\section{Summary}

We report on chemical abundances in the metal-rich cluster NGC 6253, observed with VLT/FLAMES. The original sample includes seven stars (two at the turn off, one at the clump, and four on the subgiant/red giant branch). We find the following results:

1. Membership: among the seven stars in the original sample, we considered two objects along the RGB as non-members, since we are not able to discern if their radial velocities are variable; one of the two stars at TO is a radial velocity doubtful member, but its metallicity is consistent with those of the members, therefore we include it in the final sample.

2. Metallicity: we confirm that the metallicity of NGC 6253 is much higher than solar. In particular we find an average $[\mathrm{Fe} / \mathrm{H}]=+0.39 \pm 0.08$, or $[\mathrm{Fe} / \mathrm{H}]=+0.36 \pm 0.07$ if we exclude the clump star.

3. Other elements: the abundance ratios of the $\alpha$-elements $\mathrm{Si}$, $\mathrm{Ca}, \mathrm{Ti}$, and of the Fe-peak elements $\mathrm{Cr}$ and $\mathrm{Ni}$ are solar in average, while $\mathrm{Ba}$ is enhanced, as usual for open clusters. No trends with $T_{\text {eff }}$ or $[\mathrm{Fe} / \mathrm{H}]$ are present. The clump star shows a Si-enhancement and $\mathrm{Ca}, \mathrm{Ti}, \mathrm{Cr}$ abundances lower than solar; nevertheless this result might be an artifact of the Fe scale offset. The $\alpha$-element $\mathrm{Mg}$ appears to be enhanced in all the stars, but the analysis is based only on two lines that give scattering results. Also, the abundance of the light element $\mathrm{Na}$ appears enhanced (at $[\mathrm{Na} / \mathrm{Fe}]$ about +0.2 ) if the analysis is carried out in LTE. On the other hand, if the nonLTE corrections by Mashonkina et al. (2000) are taken into account, the average $[\mathrm{Na} / \mathrm{Fe}]$ ratio becomes nearer to solar.
4. Origin of the cluster: we compared the average abundances of the explosive nucleosynthesis $\alpha$-elements $\mathrm{Si}, \mathrm{Ca}$, and $\mathrm{Ti}$ in NGC 6253 (excluding the clump star), and other stars of the Galactic population: open clusters, disk field stars, and bulge/bulge-like field stars.

- Comparisons with open clusters and field stars: $\alpha$ element abundances in NGC 6253 are similar to those of other open clusters and field disk stars with oversolar Fe content; on the other hand, field stars located towards the bulge are characterized by an $\alpha$-enhancement, with a large dispersion for $[\mathrm{Fe} / \mathrm{H}]>0$. Therefore, it seems more likely that NGC 6253 was born in the disk, in a region where a particular Fe enrichment occurred.

- Fe gradient: in the context of the radial gradient (the $[\mathrm{Fe} / \mathrm{H}]$ distribution as a function of Galactocentric radius), NGC 6253 appears to lie slightly above the other clusters with similar $R_{\mathrm{gc}}$, due to its very high $[\mathrm{Fe} / \mathrm{H}]$. Therefore, either it had a different formation history and evolution from other open clusters (but this is unlikely for NGC 6253), or the gradient is very steep for clusters located at low $R_{\mathrm{gc}}$, since the metallicities derived from high resolution spectroscopy for almost all of them are oversolar.

In summary, it is very difficult to speculate on the possible origin of NGC 6253, although a very high Fe content has been ascertained. Independent of its origin, this cluster is very interesting, and it represents an ideal target in which to search for extra-solar planets.

Acknowledgements. P.S. acknowledges support by the Italian MIUR, under PRIN 20040228979-001, and by INAF-Osservatorio Astrofisico di Arcetri, where this work was completed. We thank the anonymous referee for her/his valuable suggestions. We are grateful to C. Sneden and S. Lucatello for having provided an updated version of MOOG and for useful discussion about it; we also thank G. Carraro and L. Pasquini for helpful discussion and suggestions.

\section{References}

Alonso, A., Arribas, S., \& Martínez-Roger, C. 1996, A\&A, 313, 873 Alonso, A., Arribas, S., \& Martínez-Roger, C. 1999, A\&AS, 140, 261 Anders, E., \& Grevesse, N. 1989, GeCoA, 53, 197

Barklem, P. S., Piskunov, N., \& O’Mara, B. J. 2000, A\&A, 363, 1091

Bedin, L. R., Piotto, G., Carraro, G., King., I. R., \& Anderson, J. 2006, A\&A, 460L, 27

Bensby, T., Feltzing, S., Lundström, I., \& Ilyin, I. 2005, A\&A, 433, 185

Boesgaard, A. M., \& Friel, E. D. 1990, ApJ, 351, 467

Bragaglia, A., \& Tosi, M. 2006, AJ, 131, 1544

Bragaglia, A., Tessicini, G., Tosi, M., Marconi, G., \& Munari, U. 1997, MNRAS, 284,477

Bragaglia, A., Tosi, M., Carretta, E., et al. 2006, MNRAS, 366, 1493

Carraro, G., Villanova, S., Demarque, P., et al. 2006, ApJ, 643, 1151

Carretta, E., Bragaglia, A., Tosi, M., \& Marconi, G. 2000, in Stellar Clusters and Associations: Convection, Rotation, and Dynamos, ed. R. Pallavicini, G. Micela, \& S. Sciortino, ASPC, 198, 273

Carretta, E., Bragaglia, A., Gratton, R., \& Tosi, M. 2004, A\&A, 422, 951

Carretta, E., Bragaglia, A., Gratton, R., \& Tosi, M. 2005, A\&A, 441, 131

Castro, S. M., Rich, R., Grenon, M., Barbuy, B., \& McCarthy, J. 1997, AJ, 114, 376

De Silva, G. M., Freeman, K. C., Asplund, M., et al. 2006, AJ, 133, 1161

Friel, E. D. 1995, ARAA, 33, 381

Friel, E. D. 2006, in Chemical Abundances and Mixing in Stars in the Milky Way and its Satellites, ed. S. Randich, \& L. Pasquini, ESO Astrophysic Symposia, 24, 3

Friel, E. D., Janes, K. A., Tavarez, M., et al. 2002, AJ, 124, 2693

Friel, E. D., Jacobson, H. R., Barrett, E., et al. 2003, AJ, 126, 2372

Fulbright, J. P., McWilliam, A., \& Rich, R. M. 2006, ApJ, 636, 821

Fulbright, J. P., McWilliam, A., \& Rich, R. M. 2007, submitted [arXiv:astro-ph/0609087]

Gratton, R., \& Sneden, C. 1990, A\&A, 234, 366

Gratton, R., Carretta, E., Eriksson, K., \& Gustafsson, B. 1999, A\&A, 350, 955 
Gratton, R., Carretta, E., Claudi, R., Lucatello, S., \& Barbieri, M. 2003, A\&A, 404, 187

Gratton, R., Sneden, C., \& Carretta, E., 2004, ARA\&A, 42, 385

Gratton, R., Bragaglia, A., Carretta, E., \& Tosi, M. 2006, ApJ, 642, 462

Holweger, H., \& Müller, E. A. 1974, Sol. Phys., 39, 19

Johnson, H. L. 1966, ARA\&A, 4, 193

King, J. R., Bedin, L. R., Piotto, G., Cassisi, S., \& Anderson, J. 2005, ApJ, 130, 626

Kurucz, R. L. 1993, CD-ROM No. 9

Luck, R. E. 1994, ApJS, 91, 309

Mashonkina, L. I., Shimanskii, V. V., \& Sakhibullin, N. A. 2000, Astron. Rep., 44, 790

Mishenina, T. V., Soubiran, C., Kovtyukh, V. V., \& Korotin, S. A. 2004, A\&A, 418,551

Mochejska, B. J., Stanek, K. Z., Sasselov, D. D., et al. 2005, AJ, 129, 2856

Momany, Y., Vandame, B., Zaggia, S., et al. 2001, A\&A, 379, 436

Origlia, L., Valenti, E., Rich, M. R., \& Ferraro, F. R. 2006, ApJ, 646, 499

Pasquini, L., Avila, G., Allaert, E., et al. 2000, SPIE, 4008, 129

Pasquini, L., Randich, S., Zoccali, M., et al. 2004, A\&A, 424, 951

Paulson, D., Saar. S. H., Cochran, W. D., \& Henry, G. W. 2004a, AJ, 127, 1644
Paulson, D., Cochran, W. D., \& Hatzes, A. P. 2004b, AJ, 127, 3579

Piatti, A. E., Clarià, J. J., Bica, E., Geisler, D., \& Minniti, D. 1998, AJ, 116, 801 Pompeia, L., Barbuy, B., \& Grenon, M. 2003, ApJ, 592, 1173

Randich, S. 2007, in The Metal Rich Universe, ed. G. Israelian, \& A. Herrero, in press

Randich, S., Bragaglia, A., Pastori, L., et al. 2005, ESO Messenger, 121, 18

Randich, S., Sestito, P., Primas, F., Pallavicini, R., \& Pasquini, L. 2006, A\&A, 450,557

Sagar, R., Munari, U., \& de Boer, K. S. 2001, MNRAS, 327, 23

Santos, N. C. 2006, in Chemical Abundances and Mixing in Stars in the Milky Way and its Satellites, ed. S. Randich, \& L. Pasquini, ESO Astrophys. Symp., 24, 19

Sestito, P., Randich, S., Mermilliod, J.-C., \& Pallavicini, R. 2003, A\&A, 407, 289

Sestito, P., Bragaglia, A., Randich, S., et al. 2006, A\&A, 458, 121 (Paper I)

Soubiran, C., \& Griard, P. 2005, A\&A, 438, 139

Sneden, C. A. 1973, ApJ, 184, 839

Twarog, B. A., Anthony-Twarog, B. J., \& de Lee, N. 2003, AJ, 125, 1383

Unsöld, A. 1955, Physik der Sternatmosphären (Berlin: Springer-Verlag)

Yong, D., Carney, B. W., \& de Almeida, L. 2005, AJ, 130, 597 